\title{
WEAKLY NON-ASSOCIATIVE ALGEBRAS AND THE KADOMTSEV-PETVIASHVILI HIERARCHY
}

\author{
ARISTOPHANES DIMAKIS \\ Department of Financial and Management Engineering, \\ University of the Aegean, 31 Fostini Str., GR-82100 Chios, Greece \\ e-mail:dimakis@aegean.gr \\ and FOLKERT MÜLLER-HOISSEN \\ Max-Planck-Institute for Dynamics and Self-Organization, \\ Bunsenstrasse 10, D-37073 Göttingen, Germany \\ e-mail: folkert.mueller-hoissen@ds.mpg.de
}

\begin{abstract}
On any 'weakly non-associative' algebra there is a universal family of compatible ordinary differential equations (provided that differentiability with respect to parameters can be defined), any solution of which yields a solution of the KadomtsevPetviashvili (KP) hierarchy with dependent variable in an associative sub-algebra, the middle nucleus.
\end{abstract}

2000 Mathematics Subject Classification. 37K10 17Axx.

1. Introduction. As explained in the following, the Kadomtsev-Petviashvili (KP) hierarchy (see e.g. $[\mathbf{2}, \mathbf{1 0}]$ ) emerges from a simple algebraic problem on non-associative algebras. Let $f$ freely generate a non-associative algebra $\tilde{A}$ over a commutative ring $\mathcal{R}$ with identity element (see e.g. [11] for the algebraic structures used in this work). A derivation of $\tilde{\mathbb{A}}$ is determined by its action on the generator. A family of derivations is then obtained by choosing their action on $f$ as a non-linear homogeneous expression in $f$. The simplest choice is $\delta_{1}(f)=f^{2}$. This extends to $\tilde{A}$ via the derivation rule. For the second derivation we should choose $\delta_{2}(f)=\kappa_{1} f f^{2}-\kappa_{2} f^{2} f$ with $\kappa_{1}, \kappa_{2} \in \mathcal{R}$, since $f f^{2}$ and $f^{2} f$ are the only independent monomials cubic in $f$. Then

$$
\left[\delta_{1}, \delta_{2}\right](f)=\left(\kappa_{1}-\kappa_{2}\right) f^{2} f^{2}-\left(\kappa_{1}+\kappa_{2}\right)\left(f, f^{2}, f\right),
$$

where $(a, b, c):=(a b) c-a(b c)$ is the associator. Setting $\kappa_{1}=\kappa_{2}=1$ and passing over to $\mathbb{A}=\tilde{\mathbb{A}} / \mathcal{I}$ with the ideal generated by $\{(a, b c, d) \mid a, b, c, d \in \tilde{\mathbb{A}}\}$, which is preserved by the derivations $\delta_{1}$ and $\delta_{2}$, the latter commute on $\mathbb{A}$, and we can look for further commuting derivations. In fact,

$$
\delta_{3}(f):=f\left(f f^{2}\right)-f f^{2} f-f^{2} f^{2}+\left(f^{2} f\right) f
$$

defines another derivation which commutes with the first two. This construction can be continued ad infinitum, and the underlying general building law will be presented in Section 3. The derivations $\delta_{n}$ are subject to algebraic identities. A direct calculation reveals 
that

$$
\delta_{1}\left(4 \delta_{3}(f)-\delta_{1}^{3}(f)+6\left(\delta_{1}(f)\right)^{2}\right)-3 \delta_{2}^{2}(f)+6\left[\delta_{2}(f), \delta_{1}(f)\right] \equiv 0 .
$$

Formally replacing $\delta_{n}$ by the partial derivative $\partial_{t_{n}}$ with respect to a variable $t_{n}$, (3) becomes the potential KP equation (for $-f$, according to our convention). Elements $\delta_{n_{1}} \cdots \delta_{n_{k}}(f)$, where $n_{1}, \ldots, n_{k}=1,2, \ldots$ and $k=1,2, \ldots$, satisfy more identities of this kind, and the whole KP hierarchy emerges in this way.

If $\mathbb{A}$ is taken over a commutative ring of smooth functions of independent variables $t_{1}, t_{2}, \ldots$, we may consider the hierarchy of first-order ordinary differential equations

$$
f_{t_{n}}:=\partial_{t_{n}}(f)=\delta_{n}(f), \quad n=1,2, \ldots
$$

The first equation $f_{t_{1}}=f^{2}$, which is the only one that would survive in case of associativity, is the equation of a 'non-associative top' [8]. It has the form of a (non-associative) 'quadratic dynamical system' as considered in [9], for example. Since (4) turns the identity (3) into the potential KP equation (for $-f$ ), it follows that any solution of (4) also solves the latter. More generally, we demonstrate that this holds for any algebra $\mathbb{A}$ which is 'weakly non-associative' (WNA), and this yields solutions of the whole KP hierarchy, with dependent variable in an associative (and typically non-commutative) sub-algebra. The KP hierarchy appears in many areas of mathematics and physics, and the above result further adds to its ubiquitousness.

In Section 2 we define and characterize WNA algebras. Section 3 introduces a sequence of derived products in such algebras, which mainly serves as a preparation for the construction of a hierarchy of derivations, i.e. a sequence of commuting derivations $\delta_{n}, n=1,2, \ldots$, for a sub-class of WNA algebras. Section 4 contains a major result of this work, namely the proof that the derivations $\delta_{n}$ satisfy a sequence of algebraic identities which are in correspondence with the equations of the KP hierarchy (as outlined above). In Section 5 we derive some properties and consequences of the "non-associative hierarchy' (4). Section 6 treats a class of examples, and Section 7 contains further remarks. A preliminary account of results reported here appeared in our preprint [5], which the reader may consult as a supplement, in particular for some proofs omitted in the following.

2. Weakly non-associative algebras. Let $\mathbb{A}$ be a non-associative (and typically noncommutative) algebra over a unital commutative ring $\mathcal{R}$. The middle nucleus (see e.g. [12])

$$
\mathbb{A}^{\prime}:=\{b \in \mathbb{A} \mid(a, b, c)=0 \quad \forall a, c \in \mathbb{A}\}
$$

is then an associative sub-algebra. If $\mathbb{A}$ has an identity element, then it belongs to $\mathbb{A}^{\prime}$.

DeFINITION 2.1. $\mathbb{A}$ is called WNA if $\mathbb{A}^{2} \subset \mathbb{A}^{\prime}$, i.e.

$$
(a, b c, d)=0, \quad \forall a, b, c, d \in \mathbb{A} .
$$

If $\mathbb{A}$ is WNA, then $\mathbb{A}^{\prime}$ is a two-sided ideal in $\mathbb{A}$, and the quotient algebra $\mathbb{A} / \mathbb{A}^{\prime}$ is nilpotent of index 2. If $\mathbb{A}$ is any non-associative algebra and $\mathcal{I}$ the two-sided ideal in $\mathbb{A}$ generated by $(a, b c, d)$ for all $a, b, c, d \in \mathbb{A}$, then $\mathbb{A} / \mathcal{I}$ is a WNA algebra. The WNA condition (6) can also be expressed as $L_{a} L_{b}=L_{a b}$ or $R_{a} R_{b}=R_{b a}$ for all $a \in \mathbb{A}$ and $b \in \mathbb{A}^{\prime}$, where $L_{a}$ and $R_{a}$ denote, respectively, left and right multiplication by $a \in \mathbb{A}$. The following result characterizes WNA algebras and provides examples. 
Proposition 2.1. (1) Let $\mathcal{A}$ be an associative algebra over $\mathcal{R}, L_{i}, R_{i}: \mathcal{A} \rightarrow \mathcal{A}$, $i=1, \ldots, N$, linear maps such that $\left[L_{i}, R_{j}\right]=0$,

$$
L_{i}(a b)=L_{i}(a) b, \quad R_{i}(a b)=a R_{i}(b), \quad \forall a, b \in \mathcal{A}
$$

and $g_{i j} \in \mathbb{A}, i, j=1, \ldots, N$. For $\boldsymbol{\alpha}=\left(\alpha_{1}, \ldots, \alpha_{N}\right), \boldsymbol{\beta}=\left(\beta_{1}, \ldots, \beta_{N}\right) \in \mathcal{R}^{N}$, let us define

$$
g(\boldsymbol{\alpha}, \boldsymbol{\beta}):=\sum_{i, j=1}^{N} \alpha_{i} \beta_{j} g_{\ddot{j}}, \quad L_{\boldsymbol{\alpha}}:=\sum_{i=1}^{N} \alpha_{i} L_{i}, \quad R_{\boldsymbol{\beta}}:=\sum_{i=1}^{N} \beta_{i} R_{i} .
$$

The augmented algebra $\mathbb{A}:=\left(\bigoplus_{i=1}^{N} \mathcal{R}\right) \oplus \mathcal{A}$ becomes WNA when supplied with the product

$$
(\boldsymbol{\alpha}, a)(\boldsymbol{\beta}, b):=\left(\mathbf{0}, g(\boldsymbol{\alpha}, \boldsymbol{\beta})+L_{\boldsymbol{\alpha}}(b)+R_{\boldsymbol{\beta}}(a)+a b\right) .
$$

If, in addition, the equations

$$
\begin{aligned}
& L_{\boldsymbol{\beta}} L_{\boldsymbol{\alpha}}(a)=g(\boldsymbol{\beta}, \boldsymbol{\alpha}) a, R_{\boldsymbol{\beta}} R_{\boldsymbol{\alpha}}(a)=a g(\boldsymbol{\alpha}, \boldsymbol{\beta}), \\
& \quad R_{\boldsymbol{\gamma}}(g(\boldsymbol{\beta}, \boldsymbol{\alpha}))=L_{\boldsymbol{\beta}}(g(\boldsymbol{\alpha}, \boldsymbol{\gamma})), R_{\boldsymbol{\alpha}}(a) b=a L_{\boldsymbol{\alpha}}(b),
\end{aligned}
$$

for all $a, b \in \mathcal{A}, \boldsymbol{\beta}, \boldsymbol{\gamma} \in \mathcal{R}^{N}$, imply $\boldsymbol{\alpha}=\mathbf{0}$, then $\mathbb{A}^{\prime}=\mathcal{A}$, and $\mathbb{A} / \mathbb{A}^{\prime}$ is $N$-dimensional.

(2) Any WNA algebra $\mathbb{A}$, for which $\mathbb{A} / \mathbb{A}^{\prime}$ is finite-dimensional, is isomorphic to a WNA algebra of the type described in (1).

Proof. One easily verifies that the construction in (1) satisfies (6). Defining $f_{i}:=$ $(0, \ldots, 1,0, \ldots, 0,0)$ (with identity of $\mathcal{R}$ at the $i$ th position), (10) implies that $\left[f_{i}\right] \in \mathbb{A} / \mathbb{A}^{\prime}$, $i=1, \ldots, N$, are independent. Conversely, let $\mathbb{A}$ be WNA and $f_{i} \in \mathbb{A}, i=1, \ldots, N$, such that $\left[f_{i}\right], i=1, \ldots, N$, freely generate $\mathbb{A} / \mathbb{A}^{\prime}$. Then $g_{i j}:=f_{i} f_{j} \in \mathbb{A}^{\prime}$, and $L_{i}(a):=f_{i} a$ and $R_{i}(a):=a f_{i}$ define linear maps $\mathbb{A}^{\prime} \rightarrow \mathbb{A}^{\prime}$. The WNA property implies $\left[L_{i}, R_{j}\right]=0$ and (7) with $\mathcal{A}:=\mathbb{A}^{\prime}$. Since $\left[f_{i}\right], i=1, \ldots, N$, are independent, (10) holds. Furthermore, $\iota(a):=(\mathbf{0}, a)$ for all $a \in \mathbb{A}^{\prime}$, and $\iota\left(f_{i}\right):=(0, \ldots, 1,0, \ldots, 0,0)$ (with identity at the $i$ th position), $i=1, \ldots, N$, determines an isomorphism $\iota: \mathbb{A} \rightarrow\left(\bigoplus_{i=1}^{N} \mathcal{R}\right) \oplus \mathbb{A}^{\prime}$, where the target is supplied with the product (9).

In the following, $\mathbb{A}(f)$ denotes the sub-algebra of a WNA algebra $\mathbb{A}$ generated by an element $f$.

Proposition 2.2. $\mathbb{A}(f)$ is spanned by $f$ and products of the elements $L_{f}^{n} R_{f}^{m}\left(f^{2}\right)$, $m, n=0,1,2, \ldots$

EXAMPLE: free WNA algebra. Let $\mathcal{A}_{\text {free }}$ be the free associative algebra over $\mathcal{R}$, generated by elements $c_{m, n}, m, n=0,1, \ldots$ We define linear maps $L, R: \mathcal{A}_{\text {free }} \rightarrow$ $\mathcal{A}_{\text {free }}$ by $L\left(c_{m, n}\right):=c_{m+1, n}, R\left(c_{m, n}\right):=c_{m, n+1}$ and $L(a b)=L(a) b, R(a b)=a R(b)$. As a consequence, $c_{m, n}=L^{m} R^{n}\left(c_{0,0}\right)$. The free WNA algebra $\mathbb{A}_{\text {free }}(f)$ over $\mathcal{R}$ is then defined as the algebra $\mathcal{A}_{\text {free }}$ augmented with an element $f$, such that $f f=c_{0,0}, f a=L(a), a f=R(a)$. It is easily seen that $f \notin \mathbb{A}_{\text {free }}(f)^{\prime}$; thus $\mathbb{A}_{\text {free }}(f)^{\prime}=\mathcal{A}_{\text {free }}$, and $f$ generates $\mathbb{A}_{\text {free }}(f)$. Any other WNA algebra $\mathbb{A}\left(f^{\prime}\right)$ over $\mathcal{R}$, with a single generator, is the homomorphic image of $\mathbb{A}_{\text {free }}(f)$ by the linear map given by $f \mapsto f^{\prime}$ and $c_{m, n} \mapsto L_{f^{\prime}}^{m} R_{f^{\prime}}^{n}\left(f^{\prime 2}\right)$ (cf. proposition 2.2). The derivations defined in Section 1 are well defined on $\mathbb{A}_{\text {free }}(f)$, and the reader can check the identity (3). 
3. A sequence of products and derivations of WNA algebras. Let $\mathbb{A}$ be any (non-associative) algebra. With respect to a fixed element $f \in \mathbb{A}$ we define a sequence of products $\circ_{n}, n=1,2, \ldots$, in $\mathbb{A}$ recursively by $a \circ_{1} b:=a b$ and

$$
a \circ_{n+1} b:=a\left(f \circ_{n} b\right)-(a f) \circ_{n} b, \quad n=1,2, \ldots
$$

If $f \in \mathbb{A}^{\prime}$, then $a \circ_{n} b=0$ for $n>1$. Some properties of these products are stated below. We omit the proofs which are straightforward using induction.

PROPOSITION 3.1. Let $\mathbb{A}$ be a WNA algebra. Then the products $\circ_{n}$ only depend on the equivalence class $[f] \in \mathbb{A} / \mathbb{A}^{\prime}$ and, for all $m, n \in \mathbb{N}$ and $a, c \in \mathbb{A}$, satisfy the identities

$$
\begin{aligned}
\left(a \circ_{n} b\right) \circ_{m} c & =a \circ_{n}\left(b \circ_{m} c\right) \quad \text { if } \quad b \in \mathbb{A}^{\prime}, \\
a \circ_{m+n} c & =a \circ_{m}\left(f \circ_{n} c\right)-\left(a \circ_{m} f\right) \circ_{n} c .
\end{aligned}
$$

Next we note a general property of derivations of WNA algebras and construct a family of commuting derivations for a special class of WNA algebras.

Proposition 3.2. Any derivation $\delta$ of a WNA algebra $\mathbb{A}$ with the property $\delta(\mathbb{A}) \subset \mathbb{A}^{\prime}$ is also a derivation with respect to any of the products $\circ_{n}, n \in \mathbb{N}$.

Proof. By induction. The induction step can be formulated as follows:

$$
\begin{aligned}
& \delta\left(a \circ_{n+1} b\right)=\delta\left(a\left(f \circ_{n} b\right)-(a f) \circ_{n} b\right) \\
& =\delta(a)\left(f \circ_{n} b\right)+a\left(\delta(f) \circ_{n} b\right)+a\left(f \circ_{n} \delta(b)\right)-(\delta(a) f) \circ_{n} b-(a \delta(f)) \circ_{n} b-(a f) \circ_{n} \delta(b) \\
& =\delta(a)\left(f \circ_{n} b\right)+a\left(f \circ_{n} \delta(b)\right)-(\delta(a) f) \circ_{n} b-(a f) \circ_{n} \delta(b)=\delta(a) \circ_{n+1} b+a \circ_{n+1} \delta(b),
\end{aligned}
$$

where we used the definition (11) and also (12).

DEFINITION 3.1. We call $\mathbb{A}(f) \delta$-compatible if it admits derivations $\delta_{n}, n=1,2, \ldots$, such that

$$
\delta_{n}(f) \equiv f \circ_{n} f, \quad n=1,2, \ldots
$$

For $n=1,2,3,(14)$ reproduces the derivations considered in Section 1. Clearly, $\mathbb{A}_{\text {free }}(f)$ is $\delta$-compatible. If $\mathcal{I}$ is a two-sided ideal in $\mathbb{A}_{\text {free }}(f)$ with the property $\delta_{n}(\mathcal{I}) \subset \mathcal{I}$, $n=1,2, \ldots$, then the derivations $\delta_{n}, n=1,2, \ldots$, of $\mathbb{A}_{\text {free }}(f)$ project to derivations of $\mathbb{A}_{\text {free }}(f) / \mathcal{I}$, which is then also $\delta$-compatible.

PROPOSITION 3.3. If $\mathbb{A}(f)$ is $\delta$-compatible, the derivations $\delta_{n}, n=1,2, \ldots$, commute on $\mathbb{A}(f)$.

Proof. (13) implies $f \circ_{m} \delta_{n}(f)-\delta_{m}(f) \circ_{n} f=\delta_{m+n}(f)=f \circ_{n} \delta_{m}(f)-\delta_{n}(f) \circ_{m} f$. Hence $\delta_{m} \delta_{n}(f)=\delta_{m}\left(f \circ_{n} f\right)=\delta_{m}(f) \circ_{n} f+f \circ_{n} \delta_{m}(f)=\delta_{n}(f) \circ_{m} f+f \circ_{m} \delta_{n}(f)=\delta_{n} \delta_{m}(f)$.

4. KP identities. In this section we consider a $\delta$-compatible sub-algebra $\mathbb{A}(f)$ of a WNA algebra $\mathbb{A}$, derive identities for the elements $\delta_{n_{1}} \cdots \delta_{n_{r}}(f)$ and establish a correspondence with the equations of the (potential) KP hierarchy. Since, according to propositions 3.2 and 3.3, the $\delta_{n}$ are commuting derivations of $\mathbb{A}(f)$ with respect to all products $\circ_{k}$, the formal power series $\exp \left(\sum_{n \geq 1}\left(\lambda^{n} / n\right) \delta_{n}\right)$ with an indeterminate $\lambda$ defines 
a homomorphism. Here $\mathcal{R}$ has to be extended to the ring $\mathcal{R}[[\lambda]]$ of formal power series in $\lambda$. On $\mathbb{A}(f)$ we can now define an algebraic analogue of a Miwa shift [13],

$$
a_{ \pm[\lambda]}:=\exp \left( \pm \sum_{n \geq 1} \frac{\lambda^{n}}{n} \delta_{n}\right) a .
$$

LEMMA 4.1.

$$
h(\lambda):=\sum_{n \geq 0} \lambda^{n} L_{f}^{n}(f)=f_{[\lambda]}, \quad e(\lambda):=\sum_{n \geq 0} \lambda^{n} R_{f}^{n}(f)=f_{-[-\lambda]} .
$$

Proof. Setting $h_{n}:=L_{f}^{n}(f)$ and using (13), one first proves by induction

$$
\delta_{n}(f)=h_{n}-\sum_{k=1}^{n-1} \delta_{k}(f) h_{n-1-k}
$$

and with its help, again by induction,

$$
n h_{n}=\sum_{k=1}^{n} \delta_{k}\left(h_{n-k}\right), \quad n=1,2, \ldots
$$

In terms of $h(\lambda)$, this can be expressed as

$$
\frac{d}{d \lambda} h(\lambda)=\delta_{\lambda}(h(\lambda)), \quad \delta_{\lambda}:=\sum_{n \geq 1} \lambda^{n-1} \delta_{n},
$$

which integrates to (note that $h(0)=f$ )

$$
h(\lambda)=\exp \left(\sum_{n \geq 1} \frac{\lambda^{n}}{n} \delta_{n}\right) f=f_{[\lambda]} .
$$

The second formula in (16) can be verified in a similar way.

In terms of the elementary Schur polynomials $\mathbf{p}_{n}$ and $\tilde{\delta}:=\left(\delta_{1}, \delta_{2} / 2, \delta_{3} / 3, \ldots\right),(16)$ reads

$$
L_{f}^{n}(f)=\mathbf{p}_{n}(\tilde{\delta})(f), \quad R_{f}^{n}(f)=(-1)^{n} \mathbf{p}_{n}(-\tilde{\delta})(f), \quad n=1,2, \ldots
$$

THEOREM 4.1.

$$
\begin{aligned}
-\delta_{1}\left(f_{\left[\lambda_{1}\right]}-f_{\left[\lambda_{2}\right]}\right)= & \left(\lambda_{1}^{-1}-\lambda_{2}^{-1}+f_{\left[\lambda_{1}\right]}-f_{\left[\lambda_{2}\right]}\right)\left(f_{\left[\lambda_{1}\right]+\left[\lambda_{2}\right]}-f_{\left[\lambda_{1}\right]}-f_{\left[\lambda_{2}\right]}+f\right) \\
& +\left[f_{\left[\lambda_{1}\right]}-f, f_{\left[\lambda_{2}\right]}-f\right] .
\end{aligned}
$$

Proof. The trivial identities $L_{f}^{n+1}(f)=f L_{f}^{n}(f)$ are combined into $h(\lambda)=f+\lambda f h(\lambda)$. By use of (16) and $\delta_{1}(f)=f^{2}$, this leads to

$$
\left(\lambda^{-1}-f\right)\left(f_{[\lambda]}-f\right)=\delta_{1}(f) .
$$

We rename $\lambda$ to $\lambda_{1}$. After application of an algebraic Miwa shift with $\lambda_{2}$ and subtraction of the original equation, anti-symmetrization in $\lambda_{1}, \lambda_{2}$ eliminates terms not in $\mathbb{A}^{\prime}$ and leads to (18). 
COROLlary 4.1 .

$$
\sum_{i, j, k=1}^{3} \varepsilon_{i j k}\left(\lambda_{i}^{-1}-f_{\left[\lambda_{k}\right]}+f\right)\left(f_{\left[\lambda_{i}\right]}-f\right)_{\left[\lambda_{k}\right]}=0,
$$

where $\varepsilon_{i j k}$ is totally anti-symmetric with $\varepsilon_{123}=1$.

Proof. This follows by adding (18) three times with cyclically permuted indeterminates $\lambda_{1}, \lambda_{2}, \lambda_{3}$.

It is important to note that all terms appearing in (18) and (20) lie in the associative sub-algebra $\mathbb{A}(f)^{\prime}$. (Hence the bare $f$ 's appear only spuriously and actually drop out.) The first non-trivial identity which results from expanding these functional equations in powers of the indeterminates is (3), which has the form of the potential KP equation. In fact, formally replacing $\delta_{n}$ by the partial derivative $\partial_{t_{n}}$ with respect to a variable $t_{n}, n=1,2, \ldots$, equation (20) becomes a functional representation of the potential KP hierarchy $[\mathbf{1}, \mathbf{4}]$, and the equivalent formula (18) is turned into a non-commutative version of the differential Fay identity (cf. [4]).

REMARK. In the proof of theorem 4.1, one arrives at the same result by starting alternatively from the identities $R_{f}^{n+1}(f)=R_{f}^{n}(f) f$, which translate into

$$
\left(f_{-[\lambda]}-f\right)\left(\lambda^{-1}+f\right)=-\delta_{1}(f) .
$$

5. From a non-associative hierarchy of ODEs to the KP hierarchy. In this section, $\mathbb{A}$ denotes a WNA algebra with the property that its elements are (infinitely often) differentiable with respect to variables $\mathbf{t}=\left(t_{1}, t_{2}, \ldots\right)$. The ordinary differential equations

$$
f_{t_{n}}=f \circ_{n} f, \quad n=1,2, \ldots
$$

then constitute a 'non-associative hierarchy' according to the following proposition. We shall assume that $f \notin \mathbb{A}^{\prime}$, since otherwise (22) would reduce to a single equation. In the following, $\mathbb{K}$ stands for $\mathbb{R}$ or $\mathbb{C}$ and $\mathbb{A}(f, \mathbb{K})$ denotes the WNA algebra generated in $\mathbb{A}$ by $f \in \mathbb{A}$ with coefficients in $\mathbb{K}$.

PROPOSITION 5.1.

(1) The flows (22) commute.

(2) For any solution $f$ of $(22), \mathbb{A}(f, \mathbb{K})$ is $\delta$-compatible.

Proof. Since (22) implies $f_{t_{n}} \in \mathbb{A}^{\prime}$, it follows (cf. the proof of proposition 3.2) that the flow derivatives $\partial_{t_{n}}$ act as derivations of the products $\circ_{m}$ in $\mathbb{A}(f)$. The commutativity of the flows can now be checked directly as follows, by use of (13):

$$
\begin{aligned}
\left(f_{t_{m}}\right)_{t_{n}} & =\left(f \circ_{m} f\right)_{t_{n}}=f_{t_{n}} \circ_{m} f+f \circ_{m} f_{t_{n}}=\left(f \circ_{n} f\right) \circ_{m} f+f \circ_{m}\left(f \circ_{n} f\right) \\
& =f \circ_{n}\left(f \circ_{m} f\right)-f \circ_{m+n} f+\left(f \circ_{m} f\right) \circ_{n} f+f \circ_{m+n} f \\
& =\left(f \circ_{m} f\right) \circ_{n} f+f \circ_{n}\left(f \circ_{m} f\right)=\left(f_{t_{n}}\right)_{t_{m}} .
\end{aligned}
$$

Since $\partial_{t_{n}}$ in particular extends as a derivation to $\mathbb{A}(f, \mathbb{K})$, (22) guarantees the consistency of extending $\delta_{n}(f):=f \circ_{n} f$ to $\mathbb{A}(f, \mathbb{K})$ via the derivation property.

Now we formulate the main result of this work. 
THEOREM 5.1. Iff solves (22), then $u:=-f_{t_{1}} \in \mathbb{A}^{\prime}$ solves the KP hierarchy in $\mathbb{A}^{\prime}$.

Proof. Since $\mathbb{A}(f, \mathbb{K})$ is $\delta$-compatible by proposition 5.1 , the identity (20) holds. As a consequence of (22), the algebraic Miwa shifts can be replaced by the usual ones satisfying $f_{[\lambda]}(\mathbf{t})=f(\mathbf{t}+[\lambda])$ with $[\lambda]:=\left(\lambda, \lambda^{2} / 2, \lambda^{3} / 3, \ldots\right)$. This results in a well-known functional representation of the potential KP hierarchy $[\mathbf{1}, \mathbf{4}]$, which means that $u$ solves the KP hierarchy.

We refer to [6, 7] for exact solutions of the matrix KP hierarchy obtained with the help of this theorem. The following proposition provides us with a formal solution of the initial value problem for (22) for a subclass of WNA algebras.

Proposition 5.2. Let $\mathbb{A}$ be a WNA algebra over $\mathbb{K}[[\mathbf{t}]]$ and $f_{0} \in \mathbb{A}$ constant, $f_{0} \notin \mathbb{A}^{\prime}$, generating a $\delta$-compatible sub-algebra $\mathbb{A}\left(f_{0}, \mathbb{K}\right)$. Then

$$
f:=\mathcal{S}\left(f_{0}\right) \quad \text { with } \quad \mathcal{S}:=\exp \left(\sum_{n \geq 1} t_{n} \delta_{n}\right)
$$

(where the $\delta_{n}$ are defined in terms of $f_{0}$ ) solves the non-associative hierarchy (22).

Proof. Since the $\delta_{n}$ are commuting derivations with respect to all the products $\circ_{m}$, $m=1,2, \ldots$, the linear operator $\mathcal{S}$ on $\mathbb{A}\left(f_{0}, \mathbb{K}\right)$ is an automorphism with respect to all these products (which are defined via (11) in terms of $f_{0}$ ). Hence

$$
f_{t_{n}}=\partial_{t_{n}} \mathcal{S}\left(f_{0}\right)=\mathcal{S}\left(\delta_{n}\left(f_{0}\right)\right)=\mathcal{S}\left(f_{0} \circ_{n} f_{0}\right)=\mathcal{S}\left(f_{0}\right) \circ_{n} \mathcal{S}\left(f_{0}\right)=f \circ_{n} f .
$$

Since $\delta_{n}(f) \in \mathbb{A}\left(f_{0}\right)^{\prime}$, we have $f-f_{0} \in \mathbb{A}\left(f_{0}\right)^{\prime}$; hence $[f]=\left[f_{0}\right] \in \mathbb{A}\left(f_{0}\right) / \mathbb{A}\left(f_{0}\right)^{\prime}$. The products $\circ_{n}$ (and then also the derivations $\delta_{n}$ ) are thus equivalently defined in terms of $f$ (proposition 3.1). This proves our assertion.

The solution given by proposition 5.2 has the property

$$
f=v-\phi \quad \text { with constant } v \text { and } \quad \phi \in \mathbb{A}^{\prime} .
$$

Inserting this decomposition in (22), turns it into the Riccati-type hierarchy

$$
\phi_{t_{n}}=-v \circ_{n} v+v \circ_{n} \phi+\phi \circ_{n} v-\phi \circ_{n} \phi, \quad n=1,2, \ldots
$$

If $\phi$ solves (25), then also the potential KP hierarchy. Splitting off a constant term in (24) is natural from the point of view that the potential $\phi$ is obtained from the proper KP variable $u$ by integration with respect to $t_{1}$, so $v$ plays the role of a constant of integration.

6. A simplified case and a class of solutions of the KP hierarchy. Let $(\mathcal{A}, \circ)$ be any associative algebra over $\mathcal{R}$, and $L, R$ commuting linear maps such that $L(a \circ b)=$ $L(a) \circ b$ and $R(a \circ b)=a \circ R(b)$ for all $a, b \in \mathcal{A}$. We write $L a:=L(a)$ and $a R:=R(a)$, for short. A new associative product in $\mathcal{A}$ is then given by

$$
a \circ_{1} b:=(a R) \circ b-a \circ(L b) .
$$

Augmenting $\left(\mathcal{A}, \circ_{1}\right)$ with an element $v$ such that $v \circ_{1} v:=0, v \circ_{1} a:=L a, a \circ_{1} v:=-a R$, we obtain a WNA algebra $\left(\mathbb{A}, o_{1}\right)$ with $\mathbb{A}^{\prime}=\mathcal{A}$. Restricted to $\mathbb{A}^{\prime}$, we have $L_{v}=L$ and $R_{v}=-R$. For the products (11), defined with respect to $v$, one easily proves by induction 
that

$$
\begin{aligned}
& v \circ_{n} v=0, \quad v \circ_{n} a=L^{n} a, \quad a \circ_{n} v=-a R^{n}, \\
& a \circ_{n} b=\sum_{k=0}^{n-1}(-1)^{k}\left(R_{v}^{k} a\right) \circ_{1} L_{v}^{n-k-1} b=\left(a R^{n}\right) \circ b-a \circ L^{n} b,
\end{aligned}
$$

for all $a, b \in \mathcal{A}$. The telescoping sum in (27) is a consequence of (26). Now (25) simplifies to

$$
\phi_{t_{n}}=L^{n} \phi-\phi R^{n}+\phi \circ L^{n} \phi-\phi R^{n} \circ \phi, \quad n=1,2, \ldots
$$

According to our general results, any solution of (28) is a solution of the potential KP hierarchy in $\left(\mathcal{A}, \circ_{1}\right)$.

Now we choose $\mathcal{A}=\mathcal{A}_{\text {free }}$ (see the example in Section 2) and $\mathcal{R}=\mathbb{K}[[\mathbf{t}, \epsilon]]$. Then

$$
\delta_{n}\left(c_{r, s}\right):=L^{n} c_{r, s}-c_{r, s} R^{n}=c_{r+n, s}-c_{r, s+n}, \quad n=1,2, \ldots,
$$

determines derivations $\delta_{n}$. They extend to $\left(\mathbb{A}, \circ_{1}\right)$ by setting $\delta_{n}(v)=0$ and are derivations with respect to all products $\circ_{n}$ (proposition 3.2). For $c:=c_{0,0}$ we find

$$
\delta_{n}\left(c^{\circ m}\right)=v \circ_{n} c^{\circ m}+c^{\circ m} \circ_{n} v-\sum_{k=1}^{m-1} c^{\circ k} \circ_{n} c^{\circ(m-k)},
$$

where $c^{\circ n}$ denotes the $n$th power of $c$ using the product $\circ$. This implies

$$
\delta_{n}\left(f_{0}\right)=f_{0} \circ_{n} f_{0}, \quad \text { where } \quad f_{0}:=v-\phi_{0}, \quad \phi_{0}:=\sum_{n \geq 1} \epsilon^{n} c^{\circ n} .
$$

By proposition 5.2 and theorem 5.1,

$$
\phi=\mathcal{S}\left(\phi_{0}\right)=\sum_{n \geq 1} \epsilon^{n} \mathcal{S}(c)^{\circ n} \quad \text { with } \quad \mathcal{S}(c)=e^{\xi(\mathbf{t}, L)} c e^{-\xi(\mathbf{t}, R)},
$$

where $\xi(\mathbf{t}, L):=\sum_{n \geq 1} t_{n} L^{n}$, solves the potential KP hierarchy in $\left(\mathcal{A}_{\text {free }}, \circ_{1}\right)$. Any homomorphism $\rho$ that commutes with the partial derivatives $\partial_{t_{n}}$ induces a corresponding solution in $\rho\left(\mathcal{A}_{\text {free }}\right)$ (see also [5]).

7. Further remarks. Another possibility to derive from (19), respectively (21), an equation in $\mathbb{A}^{\prime}$, is via a decomposition (24), assuming in addition that $v a=0$, respectively $a v=0$, for all $a \in \mathbb{A}^{\prime}$. Then (22) implies $\left(\lambda^{-1}+\phi\right)\left(\phi_{[\lambda]}-\phi\right)=-\partial_{t_{1}}(\phi)$, respectively $\left(\phi_{-[\lambda]}-\phi\right)\left(\lambda^{-1}-\phi\right)=\partial_{t_{1}}(\phi)$. These are functional representations of (non-commutative) Burgers hierarchies. The simplest equations derived from them are $\phi_{t_{2}}+\phi_{t_{1} t_{1}}+2 \phi \phi_{t_{1}}=$ 0 , respectively $\phi_{t_{2}}-\phi_{t_{1} t_{1}}-2 \phi_{t_{1}} \phi=0$.

Further examples and some applications of WNA algebras in the context of KP hierarchies appeared in $[3,6,7]$. There is a WNA algebra such that (22) reproduces the Gelfand-Dickey-Sato formulation of the (potential) KP hierarchy [6]. The free WNA algebra described in Section 2 has a representation in terms of the algebra of quasisymmetric functions, which therefore also exhibits KP identities. This will be elaborated elsewhere. 
ACKNOWLEDGEMENT. This work was presented at the ISLAND 3 meeting on Islay, Scotland, in 2007. F M-H would like to thank the organizers for the invitation and financial support.

\section{REFERENCES}

1. L. V. Bogdanov and B. G. Konopelchenko, Analytic-bilinear approach to integrable hierarchies. II. Multicomponent KP and 2D Toda lattice hierarchies, J. Math. Phys. 39 (1998), 47014728. 2003).

2. L. A. Dickey, Soliton equations and Hamiltonian systems (World Scientific, Singapore,

3. A. Dimakis and F. Müller-Hoissen, A new approach to deformation equations of noncommutative KP hierarchies, J. Phys. A: Math. Theor. 40 (2007), 7573-7596.

4. A. Dimakis and F. Müller-Hoissen, Functional representations of integrable hierarchies, J. Phys. A: Math. Gen. 39 (2006), 9169-9186.

5. A. Dimakis and F. Müller-Hoissen, Nonassociativity and integrable hierarchies, nlin.SI/0601001.

6. A. Dimakis and F. Müller-Hoissen, Weakly nonassociative algebras, Riccati and KP hierarchies, nlin.SI/0701010, in Generalized Lie Theory in Mathematics, Physics and Beyond (Silvestrov S., Paal E., Abramov V. and Stolin A., Editors) (Springer, 2008) 9-27.

7. A. Dimakis and F. Müller-Hoissen, With a Cole-Hopf transformation to solutions of the noncommutative KP hierarchy in terms of Wronski matrices, J. Phys. A: Math. Theor. 40 (2007), F321-F329.

8. I. Z. Golubchik, V. V. Sokolov and S. I. Svinolupov, A new class of nonassociative algebras and a generalized factorization method, ESI preprint 53 (1993). ftp://ftp.esi.ac.at/ pub/Preprints/esi053.pdf.

9. M. K. Kinyon and A. A. Sagle, Quadratic dynamical systems and algebras, J. Diff. Equations 117 (1995), 67-126.

10. B. A. Kupershmidt, Mathematical surveys and monographs: KP or $m K P$, vol. 78 of (American Math. Society, Providence, RI, USA, 2000).

11. E. N. Kuz'min and I. P Shestakov, Non-associative structures, in Algebra VI (Kostrikin A. I. and Shafarevich I. R., Editors) (Springer, Berlin, 1995) 197-280.

12. H. O. Pflugfelder, Quasigroups and loops, vol. 7 (Heldermann, Berlin, 1990).

13. M. Sato and Y. Sato, Soliton equations as dynamical systems on infinite dimensional Grassmann manifold, in Nonlinear partial differential equations in applied science, vol. 5 (Fujita H., Lax P. D. and Strang G., Editors) (North-Holland, Amsterdam, 1982) 259-271. 